\title{
LA FORMACIÓN REFLEXIVA DEL PROFESORADO COMO MARCO DE REFERENCIA EN LA MATEMÁTICA EDUCATIVA
}

\section{THE REFLECTIVE TRAINING OF TEACHERS AS A REFERENCE FRAMEWORK IN MATHEMATICS EDUCATION}

\author{
Judith Hernández Sánchez \\ Universidad Autónoma de Zacatecas. México \\ judith700@hotmail.com \\ Angélica Dueñas Cruz \\ Benemérita Escuela Normal “Manuel Ávila Camacho”. México \\ duenascruz@gmail.com \\ Mayra A. S. Báez Melendres \\ Cinvestav-IPN. México \\ mbaez@cinvestav.mx \\ Nehemías Moreno Martínez \\ Universidad Autónoma de San Luis Potosí. México \\ nehemias_moreno@live.com
}

\begin{abstract}
RESUMEN
La reflexión como un proceso necesario en la formación y desarrollo profesional de los profesores ha ganado terreno en los últimos años. La razón es que se presenta como un medio para que los profesores reconozcan acciones o prácticas que le impiden un mejor desempeño; además de que promueve la construcción de competencias docentes. Por esta razón, se presentan algunas perspectivas acerca del paradigma de la reflexión y el papel de la práctica reflexiva en la formación y desarrollo profesional del profesor; se identifican los componentes base de los procesos reflexivos según la literatura del campo. Finalmente se presentan dos propuestas, desde la Matemática Educativa, de cómo promover una práctica reflexiva en la formación de profesores de matemáticas. La primera mediante la problematización del saber y la segunda a través del uso de mapas conceptuales híbridos. Esto evidencia opciones para mejorar la formación de futuros profesionales de la enseñanza y el aprendizaje de las matemáticas.
\end{abstract}

Palabras Clave: Desarrollo Profesional, Práctica Reflexiva, Profesores de Matemáticas.

Investigación e Innovación en Matemática Educativa (2020) Volumen 5

Recibido: Junio 25, 2019. Aceptado: Septiembre 2, 2019. Publicado: Marzo 22, 2020.

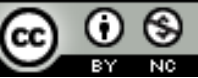

Este obra está bajo una licencia de Creative Commons Reconocimiento-NoComercial 4.0 Internacional 


\begin{abstract}
Reflection as a necessary process in the training and professional development of teachers has gained advancement in the field in recent years. The reason is that it is presented as a means for teachers to recognize those actions or practices that prevent from a better performance. In addition, promotes the construction of teaching skills. For this reason, some perspectives on the paradigm of reflection and the role of reflective practice in teacher training and professional development are presented; the base components of the reflective processes according to the literature of the field is presented. Finally, two proposals from Mathematics Education are presented, on how to promote a reflective practice in the training of mathematics teachers. The first, through the problematization of knowledge and the second through the use of hybrid concept maps. This shows options to improve the training of future professionals in the teaching and learning of mathematics.
\end{abstract}

Keywords: Professional Development, Reflective Practice, Mathematics Teachers.

\title{
1. INTRODUCCIÓN
}

La práctica docente es una actividad compleja que requiere del aprendizaje continuo y la capacidad de ser crítico; es aquí donde la práctica reflexiva podría ser una plataforma para lograr competencias profesionales. Según Villalobos y Cabrera (2009) una actitud reflexiva puede permitir al profesor reconocer acciones o prácticas que le impiden una mejor competencia docente. Además, cuando el profesor reflexiona continuamente acerca de su trabajo diario, esto puede influir significativamente en sus prácticas; en particular, asumir el control, fortalecer la toma de decisiones argumentadas y actuar para lograr cambios sobre su vida profesional. De esta forma, "los 'profesionales reflexivos' llegan a percibirse a sí mismos como agentes de cambio, capaces de comprender no sólo lo que existe, sino además esforzándose por potenciar lo que existe de manera exponencial" (Villalobos y Cabrera, 2009, p. 142).

De manera suplementaria, los resultados de algunas investigaciones consideran a la reflexión como un proceso fundamental para el desarrollo profesional docente. Principalmente porque permite estudiar aspectos de la formación inicial y continua (Climent, Romero-Cortés, Carrillo, Muñoz-Catalán y Contreras, 2013); la identidad profesional (Guichot, 2013; Walshaw, 2010); la autoevaluación y autoformación (Tzur, 2001); la investigación de la práctica (Díaz, 2006) y la profesionalización docente (Perrenoud, 2004). En otras palabras, es una herramienta para que el profesor construya conocimiento (Abkari, 2007; Dewey, 1998); en este caso, aquel que le 
permita ampliar y profundizar sobre su práctica docente y otros aspectos que de ella se derivan (Gómez-Chacón y Planchart, 2005; Sañudo, 2006).

El paradigma de la formación reflexiva (Schön, 1992; Dewey, 1998; Anijovich, Cappelletti, Mora y Sabelli, 2009; Brubacher, Case y Reagan, 2013) ha cobrado fuerza como marco de referencia en las últimas reformas curriculares para la formación inicial de profesores de matemáticas. Sin embargo, para que el profesor aprenda a reflexionar es necesario, como proponen González (2000), Flores (2007), Godino y Batanero (2009) y Alsina (2010), dar cuenta de la importancia de analizar la práctica formadora. Lo anterior, con la intención de apropiarse del paradigma reflexivo como opción para la formación de los profesores de matemáticas. Estas investigaciones respaldan nuestro interés por indagar sobre la reflexión en la formación de profesores de matemáticas y algunas propuestas o planteamientos para abordarla o promoverla desde la Matemática Educativa. Por tal motivo, el presente documento tiene lo siguientes objetivos:

- Presentar el desarrollo y algunas perspectivas acerca del paradigma de la reflexión en la formación de profesores.

- Mostrar las principales componentes que constituyen los procesos reflexivos y sugerencias de cómo promoverlos en la formación de profesores de matemáticas.

- Describir el planteamiento de dos posturas teóricas para promover la reflexión en la formación de profesores de matemáticas. El primero mediante la problematización del saber y el segundo a través del uso de mapas conceptuales híbridos.

\section{LA REFLEXIÓN EN LA FORMACIÓN DE PROFESORES}

Camilloni (2009) señala que es necesario mejorar los procesos de formación docente en lo tocante a la formación disciplinaria, pedagógica y didáctica de los profesores con la finalidad de mejorar la calidad de los aprendizajes de los alumnos y así contribuir a disminuir las brechas entre las prácticas docentes cotidianas y la formación de sujetos reflexivos. Esta distancia entre el decir y el hacer, Schön (1992) la explica a través de lo que llama "teorías en uso" y "teorías adoptadas", mismas que se encuentran mediadas por el desarrollo de habilidades del pensamiento reflexivo. De 
acuerdo con Dewey (1998), aunque no se puede enseñar a otra persona cómo pensar, sí es posible cultivar actitudes y hábitos del pensamiento que permitan hacer más corta la distancia entre lo que se dice y lo que se hace. Así, el paradigma del pensamiento reflexivo se convierte en una opción no sólo viable, sino necesario para la formación de los profesores de matemáticas.

Domingo y Gómez (2014) mencionan que la reflexión establece una posible vinculación entre la teoría y la práctica del profesor. Más aún, estas autoras afirman que “... el conocimiento profesional se produce solo cuando la práctica es una práctica pensada, cuando la reflexión hace que la práctica proyecte sobre el espejo del pensamiento para analizarla y reajustarla” (p. 3). De esta manera, reflexionar es "analizar nuestra experiencia a la luz de las evidencias recogidas y, después, valorarlas mediante el contraste con referentes pertinentes: la experiencia de otros, los avances del conocimiento, la literatura especializada, etc.” (p. 5).

La influencia fundamental sobre la reflexión en la educación se le atribuye a John Dewey. El distingue entre la acción concebida como rutina y la acción que es reflexiva, lo que lo llevó a contrastar el pensamiento crítico con los hábitos de pensamiento que no son sistemáticos, que carecen de evidencia, que se apoyan en creencias o suposiciones erróneas (Villalobos y Cabrera, 2009). Además, Dewey (1998) señala que el pensamiento requiere una cuidadosa y atenta orientación educativa, y que la necesidad de educarlo consiste en que el sujeto desarrolle sus mejores y no sus peores posibilidades. Advierte que cuando una teoría errónea logra aceptación general, los hombres emplean toda la ingenuidad de su pensamiento en reafirmarla con errores adicionales antes que renunciar a ella y comenzar en una nueva dirección. Es decir, se promueven hábitos erróneos.

La noción de práctica reflexiva fue popularizada en los Estados Unidos a principio de los años ochenta, en primer lugar por el trabajo de Donald Schön (1983, 1992), quien criticó la prevaleciente representación del docente como un técnico. Schön sustituyó esta noción, por la conceptualización del docente comprometido, responsable, autónomo en la toma de decisiones, el cual aprende continuamente y reconstruye su propia experiencia a través de la constante introversión (Villalobos y Cabrera, 2009). Esta nueva perspectiva coincide con el panorama 
presentado por Paquay, Altet, Charlier y Perrenoud (2005), donde la pregunta central es cómo formar profesionales encargados de organizar situaciones de aprendizaje más allá de reforzar sus habilidades prácticas. Es decir, esta nueva forma de ver al profesional de la enseñanza y ahora del aprendizaje propone una evolución de la profesión de profesor, estableciendo que será capaz de realizar actos intelectuales no rutinarios basados en conocimientos disciplinares y prácticas asociadas a su contexto. Para lograrlo se propone desarrollar una práctica reflexiva que atienda, entre otras necesidades, las incertidumbres relacionadas con la toma de decisiones diarias que afectan la vida de los estudiantes (Anijovich, Cappelletti, Mora, Sabelli, 2009; Villalobos y Cabrera, 2009).

En términos generales, la práctica reflexiva en la formación inicial de profesores de matemáticas apunta hacia la profesionalización de la enseñanza de las matemáticas, como una manera de contribuir a la conformación de estructuras mentales sólidas en los educandos; de manera que, tanto para el profesor como para los estudiantes, las matemáticas sean vistas como un medio para fortalecer habilidades de pensamiento. Esto permitirá en el mediano plazo que las actitudes y hábitos intelectuales se reflejen en acciones concretas evidenciando un pensamiento reflexivo. Por ejemplo, en la toma consciente y argumentada de decisiones, el análisis permanente de su práctica docente y educativa y la iniciativa para realizar adecuaciones a las actividades propuestas para la enseñanza de las matemáticas.

\section{COMPONENTES DE LOS PROCESOS REFLEXIVOS Y SU PROMOCIÓN EN LA FORMACIÓN DE PROFESORES DE MATEMÁTICAS}

A partir del análisis de la literatura se identificaron tres componentes que forman parte de los procesos reflexivos: la toma de conciencia, la construcción de conocimiento y la transformación de las acciones; se configura un nuevo esquema referencial (Vargas, 2003). La toma de conciencia resulta relevante en el proceso en tanto que "cualquier docente que quiera transformar racionalmente su práctica primero tiene que conocerla, no imaginarla ni suponerla" (Campechano, 2006, p. 73). La toma de conciencia no es así el fin de la reflexión, pero es fundamental para 
orientarla. La construcción de conocimiento alude a la promoción de un cambio de racionalidad sobre los conocimientos y acciones actuales (Perales, 2006), nuevos significados y bases epistemológicas cada vez más amplias que sustenten las consecuentes acciones. La transformación a partir de acciones fundamentadas que tengan una razón de hacer, y que impacten en el exterior e interior de la persona. Para Freire (1973) y Sennet (2009) la transformación de acciones parte de una trasformación de sí mismo. Estos tres componentes caracterizados no necesariamente son lineales en el proceso, pero sí fundamentales para desarrollar una praxis.

Para promover estos componentes se sugiere, según algunas propuestas desde la Matemática Educativa, la creación de espacios de desarrollo profesional (Climent y Carrillo, 2003), o bien comunidades de aprendizaje e indagación (Alsina, 2010). En ambos casos se coincide en que el aprendizaje sobre la práctica es una fuente necesaria para la reflexión. Pero debe ser creado por el propio profesor y no por los formadores o investigadores que luego lo transmiten. De tal manera que el profesor sea quien impregne de significado lo ocurrido en clase construyendo conocimiento y contrastando con la teoría para acciones futuras, donde los formadores e investigadores se convierten en mediadores y copartícipes de este proceso.

Según Ametller y Alsina (2017) una forma de promover este tipo de formación es el aprendizaje realista, "centrado en la conexión entre las experiencias del profesorado en la práctica y el conocimiento teórico" (p. 2059). Esta perspectiva propone instrumentos concretos para reflexionar sobre la práctica pero con un sustento teórico. En particular, Gellert (2005) propone que para el caso de la formación inicial los futuros profesores se vuelven más reflexivos si la reflexión se dirige explícitamente hacia la práctica escolar. En este mismo tenor, Planchart, Garbin y Gómez-Chacón (2005) reconocen la necesidad de profesionales reflexivos capaces de conectar teoría y práctica y de combinar en el aula de matemáticas teoría e investigación.

De esta manera la práctica reflexiva se convierte en una herramienta para promover competencias deseables en los profesores. Vilaró (2005) señala los desafíos de la formación docente ante la realidad social y la sociedad del conocimiento en el marco de cooperación internacional América Latina-Europa. Aquí se hace presente la capacidad de reflexión como parte 
del perfil de egreso deseable del futuro profesor como educador y como profesor de matemáticas. El educador será reflexivo "respecto a las relaciones entre sociedad y educación en una perspectiva histórica" (Vilaró, 2005, p. 64); mientras que el profesor de matemáticas reflexionará "respecto a todos los tópicos de matemáticas pertinentes [...] y las competencias a promover en los estudiantes..." (Vilaró, 2005, p. 64).

Por tal motivo a continuación se presentan dos planteamientos que se sustentan en enfoques teóricos de la Matemática Educativa para promover la reflexión en la formación de profesores de matemáticas, donde la Matemática Escolar se convierte en el objeto central de la reflexión.

\section{DOS PLANTEAMIENTOS PARA PROMOVER LA REFLEXIÓN EN LA FORMACIÓN DE PROFESORES DE MATEMÁTICAS}

\subsection{La problematización del saber matemático como proceso reflexivo en la postura Socioepistemológica}

Conscientes del énfasis que han puesto las investigaciones por reflexionar sobre la práctica docente, proponemos como objeto de reflexión a la matemática escolar (Báez y Farfán, 2014, 2015, 2017). La Socioepistemología tiene como eje de sus estudios el saber matemático; toma en cuenta los aspectos históricos y contextuales que dan sentido y significado a la matemática misma, en otras palabras, toma como postura ontológica y epistemológica que la matemática se construye socialmente (Cantoral, 2013). De esta forma, refiere a la matemática escolar como aquella matemática que vive dentro y fuera de la escuela, que se caracteriza por su racionalidad basada en la preexistencia del objeto matemático y que, por tanto, genera en las aulas un espacio de reproducción de conocimientos. A este fenómeno se le ha llamado desde esta postura teórica, discurso Matemático Escolar (dME) (Soto y Cantoral, 2014), caracterizado por reconocer un solo conjunto de significados, procedimientos y argumentos de los conceptos matemáticos, al tiempo que reproduce conocimientos a través de diferentes medios, como el discurso de los profesores, los libros de texto, los planes y programas de estudios. Es así que la teoría ha centrado sus investigaciones en lograr el rediseño del dME (rdME), cuya base epistemológica son las prácticas

Investigación e Innovación en Matemática Educativa (2020) Volumen 5

https://doi.org/10.46618/iime.59 
(Cantoral, 2013), donde se reconoce la diversidad de las formas de construcción y argumentación del conocimiento. Dicho tránsito entre discursos a nivel teórico, alude el cambio de una racionalidad sobre la matemática escolar, que sintetiza el tránsito de los objetos a las prácticas.

Bajo estos planteamientos teóricos, desde los cuales se configuran espacios de problematización de la matemática escolar para la interacción con profesores, se desarrollan procesos de reflexión con el conocimiento matemático ya que proponen a nivel cognitivo un cambio del patrón referencial básico (Vargas, 2003). ¿Qué caracteriza a estos procesos de reflexión? Desde la literatura se han identificado tres componentes que están inmersos en un proceso de reflexión: toma de conciencia, construcción de nuevos conocimientos y transformación. Estos componentes conformarían un modelo general de reflexión.

Tales componentes se presentan en un espacio con base socioepistemológica. Se identifica en primera instancia que tomar consciencia del dME es sustancial, lo cual es provocado por confrontaciones con el conocimiento matemático para dar acceso a otra forma de pensamiento y tratamiento; esto es, el tránsito hacia el rdME. La construcción de nuevos conocimientos matemáticos refiere a una resignificación de los mismos, donde se (re)construyen al confrontar las nociones, significados y usos que dan lugar a nuevos argumentos, modelos, procedimientos, razonamientos, orientados por una problematización del saber matemático en cuestión expresada en situaciones problema intencionadas (Montiel, 2010).

La transformación tendría una base en la configuración de nuevas acciones argumentadas en las resignificaciones, ya que la resignificación evidenciada involucra el reconocimiento de una forma y tratamiento del saber matemático que está siendo puesto en funcionamiento (Montiel, 2010). En otras palabras, dicha transformación se expresa por un cambio de relación con el conocimiento matemático (Reyes-Gasperini, 2016). Así, se considera que un modelo de reflexión en la socioepistemología estará compuesto por confrontaciones, resignificaciones y acciones argumentadas. A continuación se presentan evidencias donde se muestran estas tres componentes. 


\subsubsection{Confrontación}

En el ejercicio mostrado en la figura 1 se solicita a un profesor argumentar sobre la proporcionalidad de la gráfica. El análisis del profesor a la gráfica de la figura 1 tiene un acento sobre el comportamiento de las magnitudes.

P: ... la variación de proporcionalidad directa dice que: si una cantidad aumenta y la otra aumenta de la misma forma, así como si una cantidad disminuye y la otra disminuye de la misma manera, se trata de variación proporcional directa.

Se espera que ambas aumenten o ambas disminuyan, mientras que en la gráfica las magnitudes una aumenta y la otra disminuye. Esta definición del profesor significa que la recta que cumple con tal condición es aquella que tiene una pendiente positiva.

En la figura 2, se representó numéricamente la variación donde el argumento se mantiene dado que las magnitudes mantienen el mismo comportamiento de la gráfica: una aumenta y la otra disminuye.

P: Sí, mirando la gráfica nada más, eh, hacemos lo que también aquí en la tablita aparece, que al aumentar una cantidad la otra disminuye me dice que ya no es de proporcionalidad directa.

Sin embargo, en la figura 3, la obtención de la constante de proporcionalidad directa pone en confrontación los argumentos anteriores.

P: Pareciera que esta debiera ser de proporcionalidad directa por la constante de proporcionalidad.

Esa confrontación es la que promueve la reflexión a través de la resignificación con el conocimiento matemático, cuya atención va dando cuenta de la relación que tiene el profesor con él, como se muestra a continuación. 


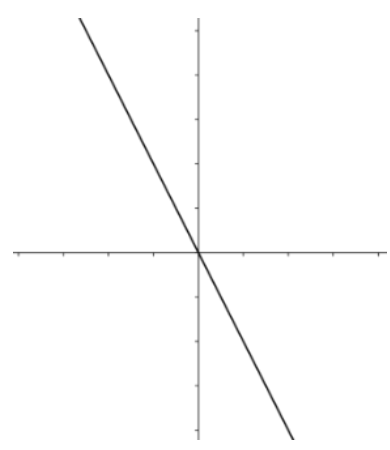

Figura 1

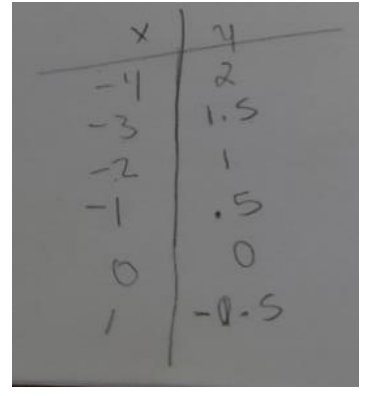

Figura 2

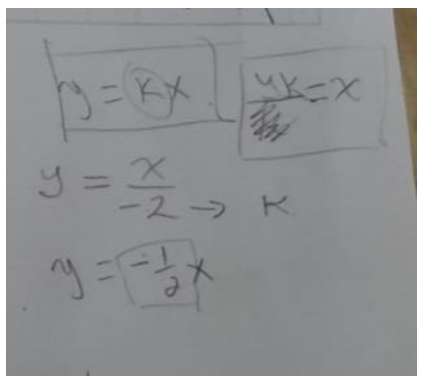

Figura 3

\subsubsection{Resignificación}

La atención a la confrontación indujo al profesor a una revisión de su estado actual del conocimiento matemático respecto de la proporcionalidad directa, lo que permitió identificar que su definición articulaba un conjunto de significados implicados, es decir, un significado tenía como consecuencia los demás:

- “de la misma manera" como ambas magnitudes aumentan o ambas disminuyen,

- la constante de proporcionalidad es válida para valores positivos,

- el paso por el origen implica proporcionalidad directa,

- el comportamiento contrario implica proporcionalidad inversa.

Particularmente el significado "de la misma manera” representaba el siguiente análisis puntual:

- un análisis separado de los valores de las magnitudes para reconocer su comportamiento cualitativo: ambas aumentan o ambas disminuyen.

- un análisis cuantitativo para comprar las magnitudes y verificar si el cambio era el mismo.

- a esto también se le llama: la relación de cantidades.

Con la atención a la confrontación, la resignificación se da como sigue:

- “de la misma manera" comienza a representar una relación de magnitudes.

- Y/X es la forma operativa de la relación. 
- La búsqueda del factor constante cobra relevancia para el análisis y argumentación de proporcionalidad directa.

Estos microscópicos cambios tienen implicaciones relevantes: que la definición inicial del profesor pierda hegemonía y sea sustituida por otra que resalta el análisis de la relación de las magnitudes, y por tanto, que el factor que subyace a esa relación sea constante; que integre los factores negativos; y que descarte que una variación contraria de las magnitudes implique proporcionalidad inversa. Así, mientras que en la confrontación el profesor da cuenta de una relación con su conocimiento matemático, en la resignificación se reconstruye, ya que sus referencias de conocimiento para el análisis, argumentación y validación se han modificado. Esta última componente se hace evidente enseguida.

\subsubsection{Acciones Argumentadas}

La resignificación anterior produce en el profesor una necesidad de modificar su práctica. Esto es, el conjunto de significados no solo son conocimiento de él, sino también aquél que enseña en su clase. Por tanto, se experimenta una necesidad de realizar cambios a la didáctica dados los recientes vividos por él respecto de la proporcionalidad directa. Estas intervenciones del profesor en el aula no formaron parte de la investigación, pero sí la continuidad de las interacciones con el profesor a través de entrevistas, lo que permitió comprobar cambios a su didáctica:

P: Ujum. Y si en algunas es fácil hallar el factor de proporcionalidad mediante estos supuestos de multiplicar un número por una variable para que nos dé la correspondiente, entonces, en el caso de que no se logra ver esto tan fácilmente, utiliza nuestras fórmulas que tenemos derivadas para poder hallar el factor de proporcionalidad, y determinar si ese factor es constante o no, ¿verdad? Si es constante entonces, lo estableceremos como de proporcionalidad directa.

En esta intervención se expresa el acento del profesor sobre el carácter constante del factor de proporcionalidad directa, que se ha vuelto referente para el análisis y la argumentación, así como conocimiento para la enseñanza. Esto evidencia de manera puntual una transformación de la práctica basada en las resignificaciones a su conocimiento matemático. Por último, es posible visualizar cómo el docente pone también un acento sobre los procedimientos, lo que indica que si 
bien ha tomado una fracción de conciencia sobre la proporcionalidad escolar y sobre significados funcionales de dicho conocimiento, el cambio del acento requerirá de procesos de reflexión permanente, es decir, de praxis.

\subsection{El Mapa Conceptual Híbrido como objeto de reflexión en el Enfoque Ontosemiótico}

El Mapa Conceptual Híbrido (MCH) es una técnica que resulta de combinar la técnica del Mapa Conceptual con la técnica del Diagrama de Flujo. En diversos trabajos (Moreno, Angulo y Reducindo, 2018; Moreno, Angulo, Reducindo y Aguilar, 2018; Moreno, Zuñiga y Tovar, 2018), se ha planteado la interpretación del $\mathrm{MCH}$ tomando en cuenta algunos elementos teóricos provenientes de la teoría del Enfoque Ontosemiótico (Godino, Batanero y Font 2007), EOS. Estos elementos son: los objetos primarios (situación-problema, lenguaje, conceptos, propiedades, procedimiento y argumentos), la función semiótica y la noción de sistema de prácticas, algunas perspectivas duales (cognitivo/epistémico, ostensivo/no-ostensivo, entre otros) así como algunos procesos cognitivos (idealización, argumentación, particularización, materialización, entre otros). En este apartado, se denota mediante MCH-EOS a la interpretación de la técnica del MCH desde el EOS. Cabe señalar que el MCH también podría ser interpretado desde otras perspectivas teóricas.

Mediante el MCH-EOS, se ha utilizado el diseño de Fislets o applets para la enseñanza de la física (Moreno, Angulo, Reducindo y Aguilar, 2018), el análisis de la resolución de problemas de Cálculo diferencial y de la Física (Moreno, 2017; Moreno, Aguilar, Angulo y Ramírez, 2019), se ha propuesto al MCH-EOS como una herramienta para investigar las concepciones de una docente y sus estudiantes sobre la noción física de marco de referencia (Hernández, 2019), se ha planteado el diseño de una herramienta gráfica para motivar el trabajo colaborativo entre los estudiantes en el aprendizaje de la cinemática (Moreno, Zúñiga y Tovar, 2018) y también se ha propuesto la reconstrucción de la práctica de resolución de problemas de física y matemáticas como ayuda para la enseñanza (Moreno, Angulo y Reducindo, 2018).

En general, los trabajos anteriores acerca del MCH-EOS podrían plantear un campo fértil para la investigación en educación matemática y en la didáctica de la física. De manera particular, 
en esta sección, se realiza la propuesta de emplear al MCH-EOS como una ayuda gráfica para la reflexión profesional de los docentes, de matemáticas o física, desde una perspectiva objetiva y crítica acerca de la actividad matemática o físico-matemática durante la clase.

Mediante esta propuesta, el MCH-EOS se configura como un instrumento que podría ayudar al profesor en la toma de conciencia, en la construcción del conocimiento y en la transformación de sus acciones en su práctica docente. Por ejemplo, para el caso de la reflexión sobre la actividad matemática en el aula por parte del profesor en formación, en la etapa de planeación de las secuencias didácticas, el docente podría elaborar un MCH-EOS para describir de manera gráfica cómo debería ser, desde la perspectiva institucional, el sistema de prácticas implicado en la resolución de un problema matemático o extra-matemático (en el contacto de la física, la química o la biología). La elaboración del MCH-EOS le permitiría considerar distintos elementos, a saber, elementos del contexto y los conocimientos previos de los alumnos, el conocimiento matemático (físico, químico o de biología para situaciones-problema extramatemáticos) y también elementos teóricos provenientes de una teoría de Matemática Educativa, el EOS.

Posteriormente, en la etapa de intervención o de implementación de las secuencias didácticas, sería posible lograr una conexión (Ametller y Alsina, 2017) entre la experiencia del docente en la clase (al abordar con los alumnos el problema matemático para el que se elaboró el MCH-EOS en la etapa de planeación) y el conocimiento que brinda el marco teórico del EOS. La comparación entre el sistema de prácticas planeado mediante el MCH-EOS y la implementación en el aula de la actividad matemática podría brindar al profesor un aprendizaje acerca de cómo mejorar su práctica docente, al conocer cuáles son las conexiones entre los objetos matemáticos que establecen los alumnos, los objetos matemáticos que los alumnos toman en cuenta (y cuáles no toman en cuenta), los procesos cognitivos que llevan o no llevan a cabo y al conocer las dificultades a las que se enfrentan los estudiantes.

A través de la evaluación y la reflexión, el profesor podría atribuir un significado a lo ocurrido en clase, ayudándole a construir conocimiento y a contrastar con la teoría para apoyarle 
en las acciones futuras. Cabe destacar que el MCH-EOS puede ser empleado como una herramienta exclusiva del profesor, tal y como se describió en los párrafos anteriores. Sin embargo, el MCHEOS también podría tener otros usos en el aula, por ejemplo, ser empleada como instrumento metodológico (Moreno, Zúñiga y Tovar, 2018), como herramienta de investigación (Moreno, Aguilar, Angulo y Ramírez, 2019) o material didáctico.

A continuación, se presenta una descripción de cómo elaborar el MCH-EOS a partir de la reconstrucción del sistema de prácticas que se presenta en un ejemplo de un libro de texto de física que describe la resolución de un problema de cinemática. Con esta descripción se pretende guiar o dar pistas al docente en la elaboración del MCH-EOS. En la figura 4 se presenta el MCH-EOS epistémico que fue reconstruido a partir de un ejemplo que se explica en un libro de texto de segundo grado de secundaria. Se trata de un sistema de prácticas conformado por tres prácticas interconectadas A, B y C e implicadas en la resolución del problema de cinemática: Usain Bolt parte del reposo y llega a una velocidad final de $44.72 \mathrm{~km} / \mathrm{h}$ en tan solo $9.58 \mathrm{~s}$. ¿Cuál será su aceleración y qué significado tiene ese valor? (Castañeda, 2013, p.45). La noción de práctica manejada en el MCH-EOS corresponde a la del EOS y es entendida como toda actuación o expresión (verbal, escrita, gráfica, etc.) realizada por alguien para resolver problemas matemáticos, comunicar a otros la solución obtenida, validarla, generalizarla (Godino, Batanero y Font, 2007)

El MCH-EOS de la figura 4 tiene como finalidad responder a una pregunta foco o problema "I", cuya respuesta inicia con la realización de la práctica A que da cuenta de la lectura e interpretación de la situación física problematizada, por ejemplo, los conceptos 1A, 6A, 13A y 14A provienen de la lectura del texto (son señalados en el texto), mientras que los argumentos 5A-9A10A, 7A-8A-13A y 5A-9A-11A que han sido materializados (no fueron señalados en ningún momento en el tratamiento de Castañeda (2013); sin embargo son supuestos de gran importancia en la física escolar) dan cuenta de la interpretación. También se consideran los conocimientos previos necesarios para entender y resolver el problema, por ejemplo, las rutas 3A-4A y 14A-15A. Esta última ruta de lectura es considerada como conocimiento previo ya que le lleva al sujeto a realizar una conversión de unidades.

Investigación e Innovación en Matemática Educativa (2020) Volumen 5 https://doi.org/10.46618/iime.59 


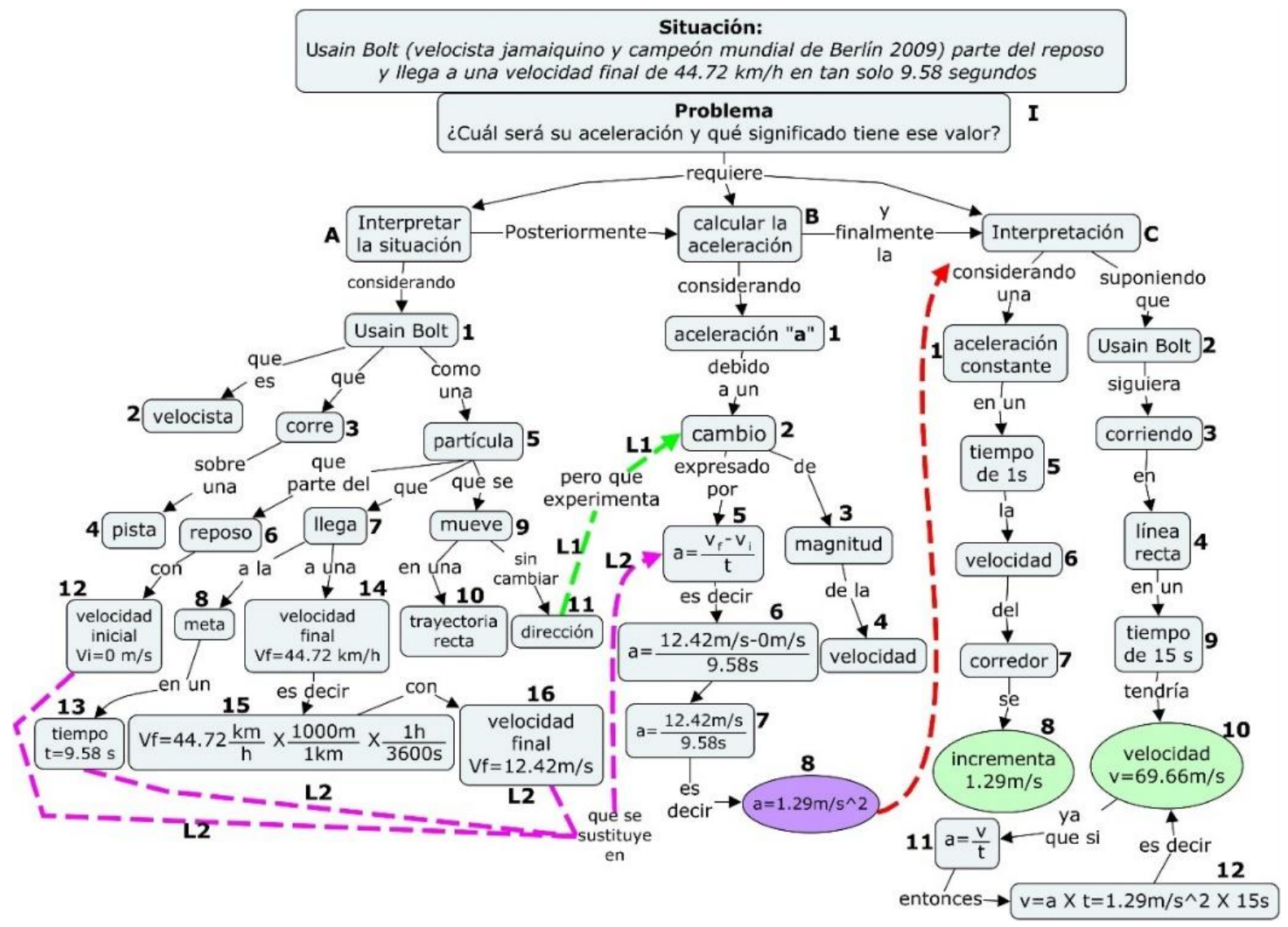

Figura 4. MCH-EOS que representa el sistema de prácticas implicado en la resolución de un problema de cinemática. Problema o pregunta focal I y sistema de tres prácticas A, B y C.

(Fuente: Elaboración propia)

Mediante la práctica B se toma en cuenta el concepto de aceleración, 1B, la propiedad del cambio en la magnitud de la velocidad, 1B-2B-3B-4B, a la que se le atribuye el significado de aceleración, la cual es representada de manera ostensiva mediante 5B. También se lleva a cabo un proceso de tratamiento físico matemático, 5B-6B-7B-8B, para calcular la aceleración del velocista, el cual inicia con la realización del proceso de particularización 5B-6B, al considerar las condiciones particulares del problema en la expresión algebraica general de la aceleración.

Y mediante la práctica $\mathrm{C}$ se interpreta el objeto unitario $8 \mathrm{~B}$ emergente de la práctica anterior, por un lado, mediante un proceso de descomposición se desmenuza a 8B en sus objetos 
componentes, cambio de velocidad 8C e intervalo de tiempo 5C, mediante el argumento $1 \mathrm{C}-5 \mathrm{C}$ 6C-7C-8C. Por otro lado, la interpretación se lleva a cabo mediante un proceso de idealización que plantea el movimiento del velocista a lo largo de una trayectoria recta durante quince segundos, C2C-3C-4C-9C, para poder generalizar el resultado para otros intervalos de tiempo advirtiendo la increíble velocidad del corredor, $10 \mathrm{C}$, mediante la realización de un tratamiento físico-matemático 10C-11C-12C. Cabe destacar también la importancia de la conexión entre las prácticas A y B, L1 y L2, que contribuye a la significación como sistema de prácticas, es decir, cada práctica tiene una finalidad particular las cuales al conectarse conducen a la solución del problema.

La reflexión sobre la práctica A por parte del docente podría ayudarle a considerar aquellos conceptos previos que son importantes para la comprensión de la situación-problema, el establecimiento de significados mediante funciones semióticas entre símbolos y conceptos (por ejemplo, "t" con el tiempo o "vf" con velocidad final). También le ayudaría a tomar en cuenta el proceso por el cual la situación-problema (pensada como situación concreta o material) es llevada al terreno de lo abstracto a través de la realización de procesos como el de idealización y particularización. Los supuestos y principios físicos considerados en esta práctica contribuirán a la interpretación del resultado $8 \mathrm{~B}$ mediante la práctica $\mathrm{C}$. La reflexión sobre la práctica $\mathrm{B}$ permite significar la aceleración en términos de la propiedad de la variación de la velocidad y significarla mediante la expresión algebraica 5B. Y finalmente, la reflexión sobre la práctica C permite al docente identificar al menos dos distintas maneras de interpretar la magnitud de la aceleración del velocista, a través de un proceso de descomposición y otra mediante un proceso de generalización.

\section{CONCLUSIONES}

Transitar hacia la formación de profesores de matemáticas reflexivos no es tarea sencilla, requiere de la participación y entendimiento de los actores involucrados en el proceso. Se trata de estar dispuestos a "mirarnos en el espejo", reconocer y modificar actitudes y hábitos que permitan reestructurar la mirada que se tiene de las matemáticas que se enseñan y la docencia. Va más allá de adecuaciones curriculares; se trata de abandonar la inmediatez, de cuestionar la normalidad y estar 
dispuestos a realizar transformaciones sustanciales que promuevan un desarrollo profesional docente.

Por tal motivo, coincidimos con autores como Perrenoud (2004) y Bazán (2008) que postulan a la reflexión como indispensable para desarrollar autonomía y responsabilidad en un profesional. Particularmente, para el docente de matemáticas, y bajo los planteamientos teóricos de la socioepistemología presentados en la sección 4.1, se busca que el proceso de empoderamiento vivenciado permita al docente el desarrollo de una autonomía sobre el conocimiento matemático y asumir la responsabilidad de su propia formación, expresadas en las acciones orientadas y sustentadas por las resignificaciones construidas. De esta forma, reconocemos que la reflexión en este terreno teórico permite al profesor cuestionar su conocimiento matemático, aspecto que trasciende a nivel didáctico y, por tanto, cuestiona también la práctica docente.

Ahora desde la propuesta ontosemiótica expuesta en la sección 4.2, el MCH-EOS podría ser empleado en las etapas (en general, las etapas de planeación, implementación y análisis) de la intervención docente; sin embargo, un objeto actual de investigación es de qué modo dicha herramienta podría ser empleada en cada etapa. En general, mediante el MCH-EOS el profesor podría orientarse sobre aspectos puntuales de la actividad físico-matemática o matemática que realiza en el aula. Dichos aspectos puntuales tienen que ver con los procesos cognitivos que son necesarios llevar a cabo y con los objetos primarios señalados por el EOS que se ponen en juego en la resolución de un problema y que son representados y conectados de manera esquemática a través del MCH-EOS. El análisis de las conexiones entre los objetos primarios podría orientar la reflexión de los docentes.

Lo anterior da evidencia de que el objeto sobre el cuál reflexiona el educador en general y el profesor de matemáticas en particular, guarda diferencias. El primero lo hace generalmente sobre el sistema educativo; mientras que el segundo tiene como eje central el contenido matemático escolar. De esta manera se justifica la necesidad de contar con mayor número de investigaciones enfocadas a la reflexión del profesor de matemáticas en particular, donde el eje rector de dicha 
reflexión es el conocimiento matemático escolar que resulte pertinente para cada nivel educativo y la promoción de los aprendizajes ligados a éstos.

\section{REFERENCIAS}

Abkari, R. (2007). Reflections on reflection: A critical appraisal of reflective practices in L2 teacher education. System, 35(2), 192-207.

Alsina, A. (2010 ). El aprendizaje reflexivo en la formación inicial del profesorado: un modelo para aprender a enseñar matemáticas. Educación Matemática, 22(1), 149-166.

Ametller, J. y Alsina, A. (2017). ¿Qué aportan el aprendizaje reflexivo y la enseñanza dialógica a la formación permanente? Un primer análisis del profesorado de ciencias y de matemáticas. Enseñanza de las Ciencias, No.Extraordinario, 2059-2064.

Anijovich, R., Cappelletti, G., Mora, S., y Sabelli, M. J. (2009). Transitar la formación pedagógica. Dispositivos y estrategias. Buenos Aires, Argentina: Paidos SAICF.

Báez, M. y Farfán, R. (2014). El rediseño de situaciones de aprendizaje y la reflexión docente. En P. Lestón (Ed.), Acta Latinoamericana de Matemática Educativa (pp. 1585-1592). México: CLAME.

Báez, M. y Farfán, R. (2015). La matemática escolar como objeto de reflexión docente. Aspectos para su desarrollo. Memorias del Vigésimo noveno de la Sociedad Española de Investigación en Educación Matemática. Alicante, España.

Báez, M. y Farfán, R. (2017). Reflexionar sobre la matemática escolar. Una ruta socioepistemológica. En L. Serna (Ed.), Acta Latinoamericana de Matemática Educativa (pp. 1037-1045). México: CLAME

Bazán, D. (2008). Autonomía profesional y reflexión docente. En D. Bazán, El oficio del pedagogo. Aportes para la construcción de una práctica reflexiva en la escuela (pp. 93-117). Rosario, Argentina: Homo Sapiens Ediciones.

Brubacher, J. W., Case, C. W., y Reagan, T. G. (2013). Cómo ser un docente reflexivo. La construcción de una cultura de la indagación en las escuelas . México : Gedisa.

Camilloni, A. (2009). Prólogo. En R. Anijovich, G. Cappelletti, S. Mora y M. (2009). Transitar la formación pedagógica. Dispositivos y estrategias (pp. 13-20). Buenos Aires, Argentina: Paidos SAICF.

Campechano, (2006). Elementos para interpretar los significados de las acciones en las prácticas educativas. En R. C. Perales (coord.), La significación de la práctica educativa (pp. 19-53). México: Paidós.

Cantoral, R. (2013). Teoría Socioepistemológica de la Matemática Educativa. Estudios sobre

Investigación e Innovación en Matemática Educativa (2020) Volumen 5

https://doi.org/10.46618/iime.59

Red de Centros de Investigación en Matemática Educativa AC 
construcción social de conocimiento. Mexico: Gedisa.

Castañeda, L. R. (2013). Ciencias 2, Física. México: Editorial Santillana S. A. de C. V.

Climent, N. y Carrillo, J. (2003). El dominio compartido de la investigación y el desarrollo profesional. Una experiencia en matemáticas con maestras. Enseñanza de las Ciencias, 21(3), 387-404.

Climent, N., Romero-Cortés, J., Carrillo, J., Muñoz-Catalán, M. y Contreras, L. (2013). ¿Qué conocimientos y concepciones movilizan futuros maestros analizando un video de aula? Revista Latinoamericana de Investigación en Matemática Educativa, 16 (1), 13-36.

Dewey, J. (1998). Cómo pensamos. Nueva exposición de la relación entre el pensamiento pensamiento reflexivo y proceso educativo . Barcelona, Buenos Aires, México: Paidos.

Díaz, V. (2006). Formación docente, práctica pedagógica y saber pedagógico. Laurus, 12(Ext), 88103.

Domingo, A. y Gómez, M. V. (2014). La práctica reflexiva. Bases, modelos e instrumentos. Madrid, España: Narcea, S. A. de Ediciones

Flores, P. (2007). Profesores de matemáticas reflexivos: formación y cuestiones de investigación. Revista de la Universidad de Granada, 1(4), 139-159.

Freire, P. (1973). Pedagogía del oprimido. (11ª edición). México: Siglo XXI Editores

Gellert, U. (2005). La formación docente entre lo teórico y lo práctico. En I. Gómez-Chacón y Planchart, E. (eds). Educación Matemática y Formación de Profesores. Propuestas para Europa y Latinoamérica (pp. 73-83). Bilbao, España: Universidad de Deusto.

Godino, J., y Batanero, C. (2009). Versión ampliada del Acta de Conferencia Invitada al VI CIBEM. (págs. 4-9). Puerto Montt (Chile): CIBEM.

Godino, J. D., Batanero, C., y Font, M. V. (2007). The onto-semiotic approach to research in mathematics education. ZDM-The International Journal on Mathematics Education, 39 (1-2), 127-135.

Gómez-Chacón, I. y Planchart, E. (2005). Educación Matemática y Formación de Profesores. Propuestas para Europa y Latinoamérica. Bilbao, España: Universidad de Deusto.

González, F. (2000). Un modelo didáctico para la formación incial de profesores de metemáticas. Sapiens. Revista Universitaria de Investigación, 11(1), 47-59.

Gellert, U. (2005). La formación docente entre lo teórico y lo práctico. En I. Gómez-Chacón y Planchart, E. (eds). Educación Matemática y Formación de Profesores. Propuestas para Europa y Latinoamérica (pp. 73-83). Bilbao, España: Universidad de Deusto.

Guichot, V. (2013). La capacidad reflexiva, factor esencial de la identidad profesional del profesorado: Reflexiones en torno a las propuestas de John Dewey y Martha Nussbaum. Cuestiones Pedagógicas, 2012-2013(22), 183-202.

Investigación e Innovación en Matemática Educativa (2020) Volumen 5

https://doi.org/10.46618/iime.59 
Montiel, G. (2010). Hacia el rediseño del discurso: formación docente en línea centrada en la resignificación de la matemática escolar. Revista Latinoamericana de Investigación en Matemática Educativa, 13(4-I), 69-84.

Moreno, M. N. (2017). Una representación gráfica de la práctica de resolución de problemas en Cálculo diferencial. Revista internacional de investigación e innovación educativa, 2017(92), 58-75.

Moreno, M. N., Aguilar, T. M. F., Angulo, V. R. G. y Ramírez, M. J. C. (2019). Análisis de la resolución de problemas de hidrostática en el bachillerato. REEC: Revista electrónica de enseñanza de las ciencias, 18(1), 274-296.

Moreno, M. N., Angulo, V. R. G., Reducindo, R. I. y Aguilar, P. R. M. (2018). Enseñanza de la física mediante fislets que incorporan mapas conceptuales híbridos. Apertura. Revista de innovación educativa, 10(2), 20-35.

Moreno, M. N., Angulo, V. R. G. y Reducindo, R. I. (2018). Mapas Conceptuales Híbridos para la enseñanza de la física y matemática escolar. Revista de Innovación e Investigación en Matemática Educativa, 3(1), 113-130.

Moreno, M. N., Zúñiga, M. S. C. y Tovar, R. D. A. (2018). Una herramienta gráfica para la resolución de problemas de cinemática. Latin American Journal of Physics Education, 12(4), 4307 1-12.Hernández, Z. L. E. (2019). Un estudio acerca de las concepciones de la noción física de marco de referencia en ingeniería. Tesis de Licenciatura no publicada. Universidad Autónoma de San Luis Potosí, San Luis Potosí, México. http://tiny.cc/byp44y

Paquay, L., Altet, M., Charlier, E. y Perrenoud, P. (2005). La formación profesional del maestro. Estrategias y Competencias. México: Fondo de Cultura Económica

Perales, R. (2006). La significación de la práctica educativa. México: Paidós.

Perrenoud, P. (2004). Desarrollar la práctica reflexiva en el oficio de enseñar. España: Graó.

Planchart, E., Garbin, S. y Gómez-Chacón, I. (2005). La Enseñanza de la Matemática en Venezuela, programa de Didáctica de la Matemática para Educación Media. En I. GómezChacón y Planchart, E. (eds). Educación Matemática y Formación de Profesores. Propuestas para Europa y Latinoamérica (pp. 33-50). Bilbao, España: Universidad de Deusto.

Reyes-Gasperini, D. (2016). Empoderamiento docente desde una visión socioepistemológica: Una alternativa para la transformación y la mejora educativa. Tesis doctoral inédita. Centro de Investigación y de Estudios del Instituto Politécnico Nacional. Cinvestav, México.

Sañudo, L. E. (2006). El proceso de significación de la práctica como sistema complejo. En R. C. Perales (coord.), La significación de la práctica educativa, 19-53. México: Paidós.

Schön, D. A. (1983). The reflective practitioner: how professionals think in action. New York: Basic Books.

Schön, D. A. (1992). La formación de profesionales reflexivos, hacia un nuevo diseño de la 
enseñanza y el aprendizaje en las profesiones. España: Paidós.

Sennet, R. (2009). El artesano. Barcelona: Anagrama.

Soto, D. y Cantoral, R. (2014). Discurso Matemático Escolar y Exclusión. Una visión

Socioepistemológica. Boletim de Educação Matemática, 28(50), 1525-1544.

Tzur, R. (2001). Becoming a mathematics teacher-educator: Conceptualizing the terrain through self-reflective analysis. Journal of Mathematics Teacher Education, 4(4), 259-283.

Vargas, Z. (2003). La confrontación: una oportunidad para el desarrollo personal. Revista Educación, 27(2), 79-86.

Vilaró, R. (2005). Desafíos de la formación docente ante la realidad social y la sociedad del conocimiento. En I. Gómez-Chacón y Planchart, E. (eds). Educación Matemática y Formación de Profesores. Propuestas para Europa y Latinoamérica (pp. 51-72). Bilbao, España: Universidad de Deusto.

Villalobos, J., y Cabrera de, C. M. (2009). Los docentes y su necesidad de ejercer una práctica reflexiva. Teoría y Didáctica de las Ciencias Sociales, 2009(14) , 139-166.

Walshaw, M. (2010). Mathematics pedagogical change: rethinking identity and reflective practice. Journal of Mathematics Teacher Education, 13(6), 487-497. DOI: 10.1007/s10857-010-91637

Investigación e Innovación en Matemática Educativa (2020) Volumen 5

https://doi.org/10.46618/iime.59 